\title{
Ear Biometrics Based on Geometrical Feature Extraction
}

\author{
Michał Choraś \\ * Institute of Telecommunication, \\ University of Technology and Agriculture, ATR Bydgoszcz, Poland
}

Received 20 December 2004, accepted 4 May 2005

\begin{abstract}
Biometrics identification methods proved to be very efficient, more natural and easy for users than traditional methods of human identification. In fact, only biometrics methods truly identify humans, not keys and cards they posses or passwords they should remember.

The future of biometrics will surely lead to systems based on image analysis as the data acquisition is very simple and requires only cameras, scanners or sensors. More importantly such methods could be passive, which means that the user does not have to take active part in the whole process or, in fact, would not even know that the process of identification takes place. There are many possible data sources for human identification systems, but the physiological biometrics seem to have many advantages over methods based on human behaviour. The most interesting human anatomical parts for such passive, physiological biometrics systems based on images acquired from cameras are face and ear. Both of those methods contain large volume of unique features that allow to distinctively identify many users and will be surely implemented into efficient biometrics systems for many applications.

The article introduces to ear biometrics and presents its advantages over face biometrics in passive human identification systems. Then the geometrical method of feature extraction from human ear images in order to perform human identification is presented.
\end{abstract}

Key Words: Biometrics, Human Identification, Computer Vision, Image Analysis, Pattern Recognition.

\section{Introduction.}

Personal identification has lately become a very important issue in a still evolving network society. Most of the traditional identification methods, which are widespread in the commercial systems, have very many disadvantages. Well known methods like entering Personal Identification Number (PIN), typing logins and passwords, displaying identification cards or using specific keys require users to take active part in the process of identification. Moreover, those traditional methods are unreliable because it is hard to remember all the PIN-s and passwords, and it is fairly easy to loose ID cards and keys. The other drawback is the lack of security, as the cards and keys are often stolen, and passwords can be cracked.

Correspondence to: < chorasm@atr.bydgoszcz.pl >

Recommended for acceptance by $<$ Perales F. Draper B. $>$

ELCVIA ISSN: 1577-5097

Published by Computer Vision Center / Universitat Autonoma de Barcelona, Barcelona, Spain 
Biometrics methods easily deal with those problems since users are identified by who they are, not by something they have to remember or carry with them. The passive methods of biometrics do not require any action from users and can take place even without their knowledge.

There are many known methods of human identification based on image analysis. In general, those biometrics methods can be divided into behavioural and physiological regarding the source of data, and can be divided into passive and invasive biometrics, regarding the way the data is acquired (Figure 1).

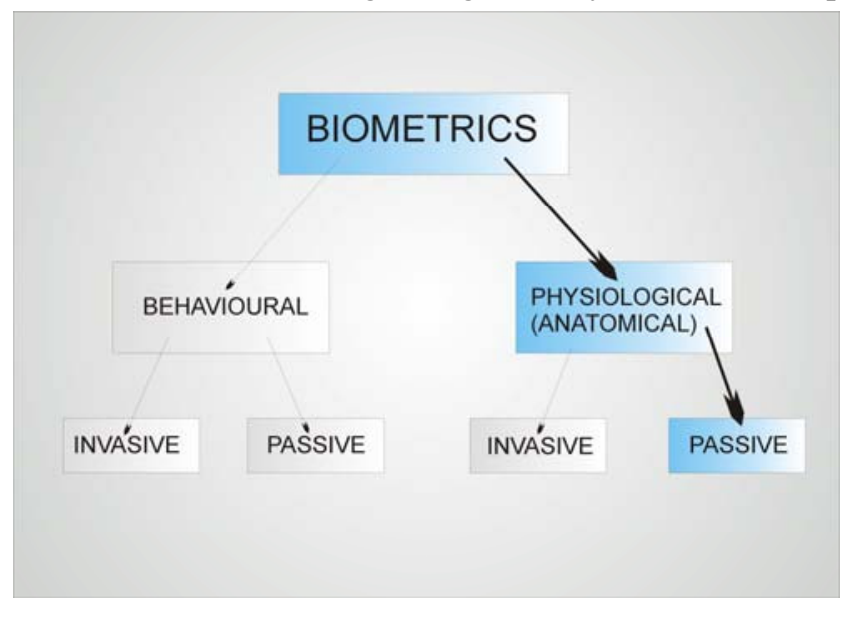

Fig. 1. Biometrics methods.

The first class is based on the behavioural features of human actions and it identifies people by how they perform something. The most popular of such methods is voice verification. Other methods are basically based on the dynamics of specific actions like making the signature, typing on the keyboard and simply moving or walking. Those methods are not that natural and they require users to take part in the process of identification by repeating specific actions, every time they are examined.

Physiological (anatomical) biometrics methods are based on the physiological features of humans thus they measure and compare features of specific parts of human body in the process of identification. So far the main interest is in the head and the hand with face, eye and fingerprint features being the most important discriminants of human identity.

The major advantage of physiological biometrics is that it is passive and the implemented systems work only with the acquired images of specific body parts. All the user has to do is to place his/her face or ear in front of the camera or alternatively touch the sensor with his/her fingers and wait for the identification process to take place.

Some systems can even verify the identity of humans even without their cooperation and knowledge, which is actually the future of biometrics. Crowd-surveillance, monitoring of public places like airports or sports arenas are the most important applications that need such solutions. Possible passive methods include popular and well-examined face recognition, but one of the most interesting novel approaches to human passive identification is the use of ear as the source of data [7].

\section{Ear Biometrics.}

Human ears have been used as major feature in the forensic science for many years. Recently so called earprints, found on the crime scene, have been used as a proof in over few hundreds cases in the Netherlands and the United States [12]. However, still the automated system of ear recognition hasn't been implemented even though there are many advantages of using ear as a source of data for person identification.

Firstly, ear does not change considerably during human life, and face changes more significantly with age than any other part of human body. Face can also change due to cosmetics, facial hair and hair styling. Secondly, face changes due to emotions and expresses different states of mind like sadness, happiness, fear or surprise. In contrast, ear features are relatively fixed and unchangeable [14]. 
Moreover, the colour distribution is more uniform in ear than in human face, iris or retina. Thanks to that fact, not much information is lost while working with the greyscale or binarized images, as we do in our method.

Figure 2 presents two more aspects of ear identification. Firstly, ear is one of our sensors, therefore it is usually visible (not hidden underneath anything) to enable good hearing. Ear is also smaller than face, which means that it is possible to work faster and more efficiently with the images with the lower resolution.

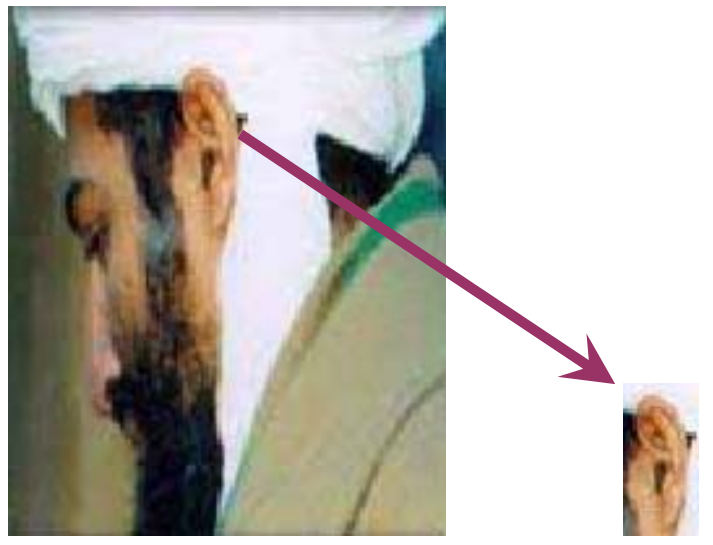

Fig. 2. Ear visibility and size.

In the process of acquisition, in contrast to face identification systems, ear images cannot be disturbed by glasses, beard nor make-up. However, occlusion by hair or earrings is possible, but in access control applications, making ear visible is not a problem for user and takes just single seconds (Figure 3).
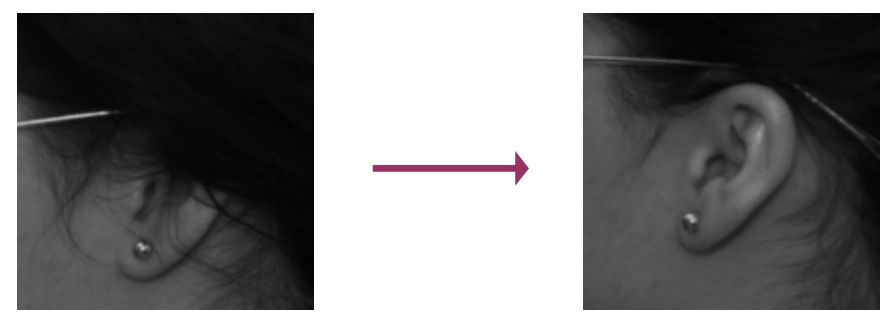

Fig. 3. Ear visibility can be easily achieved in applications allowing interaction with the user (for example access control systems)

The first, manual method, used by Iannarelli in the research in which he examined over 10000 ears and proved their uniqueness, was based on measuring the distances between specific points of the ear [14]. The major problem in ear identification systems is discovering automated method to extract those specific, key points. Another well-known method by Burge and Burger [4,5] was based on building neighbourhood graph from Voronoi diagrams of the detected edges. Hurley et al. [13] introduced a method based on energy features of the image. They proposed to perform force field transformation in order to find energy lines, wells and channels. Another method used by Victor at al. [22], in the experiment comparing ear and face properties in order to successfully identify humans in various conditions, was based on PCA. Their work proved that ear images are a very suitable source of data for identification and their results for ear images were not significantly different from those achieved for face images. The method, however, was not fully automated, since the reference points had to be manually inserted into images. Another approach presented by Moreno et al. [20] was based on macrofeatures extracted by compression networks.

\section{Geometrical Method of Feature Extraction.}

We propose a straightforward method to extract features needed to classification. Our method represents the geometrical approach, but it is automated and no manual operations are needed. After experiments we 
concluded that geometrical features representing shapes of ear contours are more suitable for ear images than texture, colour or global features.

Our method is divided into image normalization, contour extraction (edge detection), calculation of the centroid, coordinates normalization and 2 steps of geometrical feature extraction, as described in the next section. We treat the centroid as the specific point in our method, even though it is not a specific point within the ear topology.

Our method consists of the following steps (Figure 4):

- contour detection,

- binarization,

- coordinates normalization,

- feature extraction (2 steps),

- classification.

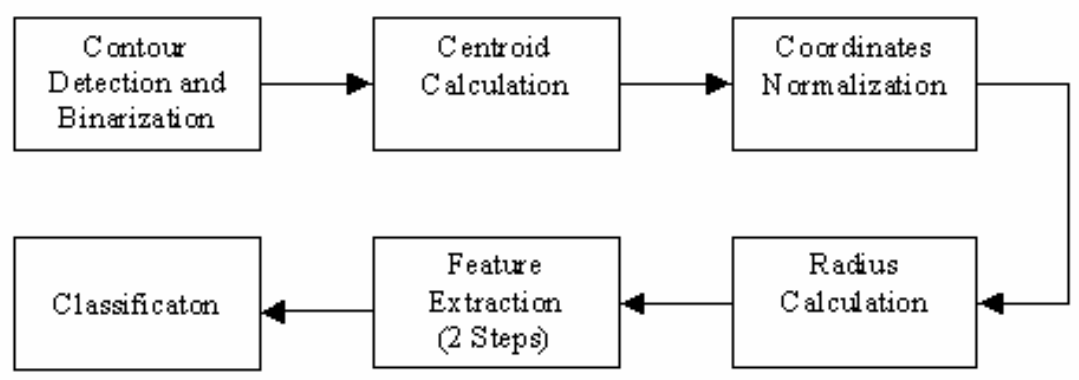

Fig. 4. The block diagram of our method.

\subsection{Contour Detection}

First we perform the edge detection step. In our case it is a crucial operation, since it is obvious that lines are the most prominent features that could be obtained from the ear image, and our goal is to detect major outer and inner curves of the earlobe. We tested many known methods as Canny operator, Sobel filters and CWT (Complex Wavelets) but we propose another method, which proved to be most convenient in our experiments.

We propose to use the local method which examines illumination changes within the chosen window $n \times n$. We usually use $3 \times 3$ window and we divided the image into many overlapping regions of that size.

For each of those regions we calculated mean $\mu$ and standard deviation $\sigma$ of the pixel intensity values in 8-pixel neighbourhood.

$$
\begin{aligned}
& \mu=\frac{1}{n^{2}} \sum_{i=1}^{n} \sum_{j=1}^{n} I(i, j) \\
& \sigma=\sqrt{\frac{1}{n^{2}} \sum_{i=1}^{n} \sum_{j=1}^{n}(I(i, j)-\mu)^{2}}
\end{aligned}
$$

Then we perform decision if the centre pixel of the examined region belongs to the line or to the background. For the maximum value of the pixel intensity $I_{H}$, and the minimum value of the pixel intensity in the region $I_{L}$, we calculate the difference $S(i, j)$ such as: 


$$
S(i, j)=I_{H}-I_{L}
$$

and we compare it to certain threshold value. Even though thresholding is one of the basic operations of image processing, there is always the major problem in selecting appropriate threshold value.

We propose the usage of mean and standard deviation of pixel intensities in calculation of the threshold value $T(i, j)$ used in contour detection as given in equation 4 :

$$
T(i, j)=\mu-k \sigma
$$

where $k$ is a certain value.

Then the rule for the contour detection is:

$$
g(i, j)=\left\{\begin{array}{lll}
1 & \text { if } \quad & S(i, j) \geq T(i, j) \\
0 & \text { if } \quad & S(i, j)<T(i, j)
\end{array}\right.
$$

In result we obtain the binary image $g(i, j)$ with the detected contours. Moreover, the constant $k$ allows to adjust and change the sensivity of the edge detection algorithm [9].

An example of the edge detection algorithm is shown in the Figure 5.
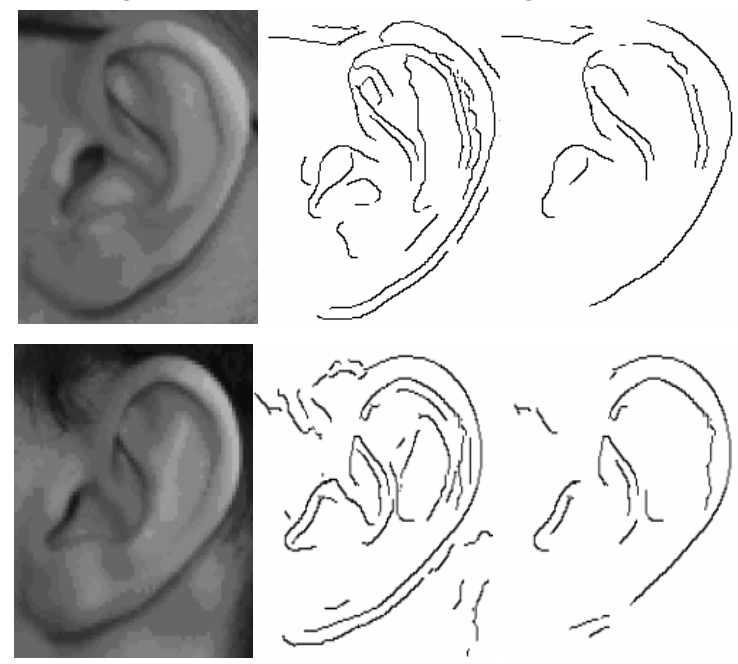

Fig. 5. Result of edge detection algorithm for two different values of $k$.

\subsection{Normalization}

Given the binary image $g(i, j)$, we search for the centroid which later becomes the reference point for feature extraction. We obtain centroid such as:

$$
I=\frac{\sum_{i} \sum_{j} i g(i, j)}{\sum_{i} \sum_{j} g(i, j)}, \quad J=\frac{\sum_{i} \sum_{j} j g(i, j)}{\sum_{i} \sum_{j} g(i, j)}
$$


Because the features for a recognition algorithm should be invariant to ear translation and scale change, the coordinates normalization is performed. Therefore we normalize coordinates, such that the centroid becomes the centre of the image. Suppose that the image with pixel coordinates $(i, j)$ undergoes geometric transformations to produce an invariant image with coordinates $(x, y)$. This transformation may be expressed as:

$$
[x, y, z]=[i, j, 1]\left[\begin{array}{ccc}
1 & 0 & 0 \\
0 & 1 & 0 \\
-I & -J & 1
\end{array}\right]\left[\begin{array}{ccc}
\frac{1}{\sigma_{i}} & 0 & 0 \\
0 & \frac{1}{\sigma_{j}} & 0 \\
0 & 0 & 1
\end{array}\right]\left[\begin{array}{ccc}
\cos \beta & \sin \beta & 0 \\
-\sin \beta & \cos \beta & 0 \\
0 & 0 & 1
\end{array}\right]
$$

where:

I, J - centroid,

$\sigma_{i}, \sigma_{j}$ - standard deviation of $i$ and $j$ respectively:

$$
\sigma_{i}=\sqrt{\frac{\sum_{i} \sum_{j} i^{2} g(i, j)}{\sum_{i} \sum_{j} g(i, j)}-I^{2}}, \quad \sigma_{j}=\sqrt{\frac{\sum_{i} \sum_{j} j^{2} g(i, j)}{\sum_{i} \sum_{j} g(i, j)}-J^{2}}
$$

Furthermore, our method is also invariant to rotation as all the rotated images of the same object have the same centroid. That is the major reason that we chose the centroid of the image to be the reference point in the feature extraction algorithm. Such approach allows the successful processing of RST queries.

\subsection{Feature extraction}

There are many possible geometrical methods of feature extraction and shape description such as Fourier Descriptors, Delaunay Triangles and methods based on combination of angles and distances as parameters. We propose a 2 step-method that is based on number of pixels that have the same radius in a circle with the centre in the centroid and on the contour topology. The algorithm for the first step of feature extraction is presented below:

1. we create a set of circles with the centre in the centroid (Figure 6)

2. number of circles $N_{r}$ is fixed and unchangeable

3. we create circles in such a manner that the corresponding radiuses are $\alpha$ pixels longer from the previous radius

4. since each circle is crossed by the contour image pixels we count the number of intersection pixels $l_{r}$

5. next we calculate all the distances $d$ between neighbouring pixels, we proceed in the counterclockwise direction

6. we build the feature vector that consists of all the radiuses with the corresponding number of pixels belonging to each radius and with the sum of all the distances between those pixels $\sum d$ 

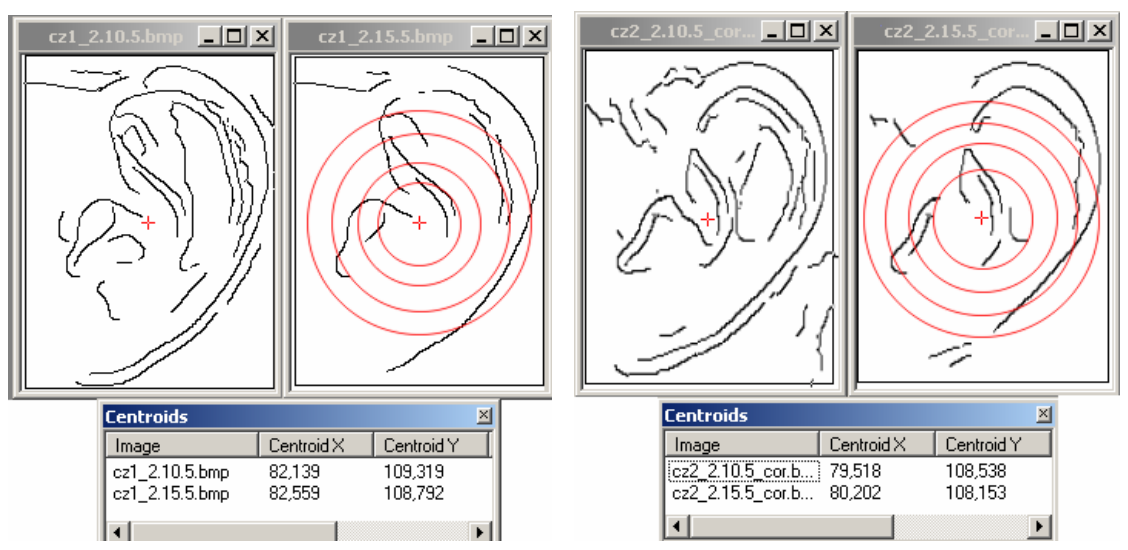

Fig. 6. Binary ear images with the extracted edges ( 2 values of $k$ ) and with the centroid marked with a cross. Circles represent the radius values for calculation of number of pixels intersecting each circle. The table below shows the centroid values for each binary image.

The algorithm for $N r=3$ is symbolically presented in the Figure 7 .

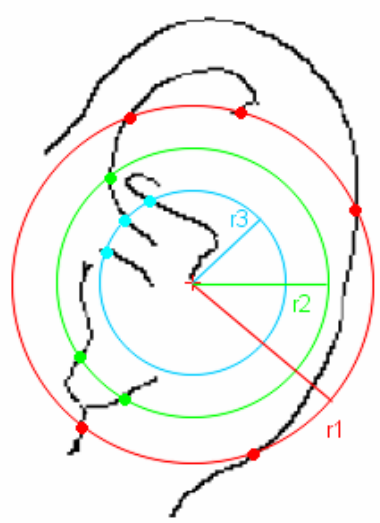

Fig. 7. The symbolic representation of our algorithm for $N_{r}=3$.

The general rule for forming the first vector is presented below:

$$
V=\left\{\left[r_{\min }, l_{r \min }, \sum d_{r \min }\right] \cdots\left[r_{\max }, l_{r \max }, \sum d_{r \max }\right]\right\}
$$

where:

$r$ - radius length,

$l_{r}-$ number of intersection points for each radius,

$\sum d$-sum of all the distances between the intersection points for the considered radius.

Then in order to enhance the distinctiveness of the extracted features, we build the second vector in the second step of feature extraction. Once again we base upon the created circles with the centre in the centroid. Hereby, we propose to extract the characteristic points for each contour in the normalized coordinates. 
For each contour line the characteristic points are:

- contour endings,

- contour bifurcations,

- all the points that cross the created circles (those points are already extracted by the previous algorithm).

In each contour we check the topological properties of every pixel. For each contour pixel $g_{o}$ we use $3 \times 3$ window as in Figure 8 (left). When $g_{0}=1$, the connected number $N_{c}^{8}$ of $g_{0}$ is defined as:

$$
N_{c}^{8}\left(g_{o}\right)=\sum_{k=S}\left(\begin{array}{c}
- \\
g_{k}-g_{k}
\end{array} g_{k+1}^{-} g_{k+2}^{-}\right)
$$

where $S=(1,3,5,7)$ and $g_{k}^{-}=g_{k}-1$
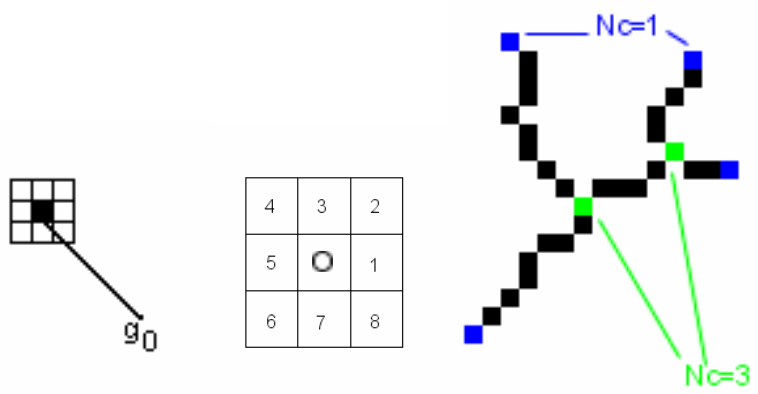

Fig. 8. Characteristic points detection algorithm.

We search for the contour beginning in the area A, such that: $r_{i} \geq A>r_{i-1}$. We begin the search for $r_{i}=r_{\max }$, which means that we start our algorithm in the most outer circle. Then we search in all other circles heading towards the centre in the centroid. If we come across any point with $N_{c}^{8}=1$, we check if it is already stored in the feature vector and if not, we store it as the ending point and we trace its contour. Points with $N_{c}^{8}=1$ and $N_{c}^{8}>2$ are the ear contour endings and the contour bifurcation points respectively. Those points are marked as $E$ and $B$ in the Figure 9.

For each contour we also extract the intersections with the circles created earlier. For each contour intersecting the circles we store all the intersections coordinates $i$ and the number of such intersections $N_{I}$ as presented in Figure 9 (right) and Eq. 11.
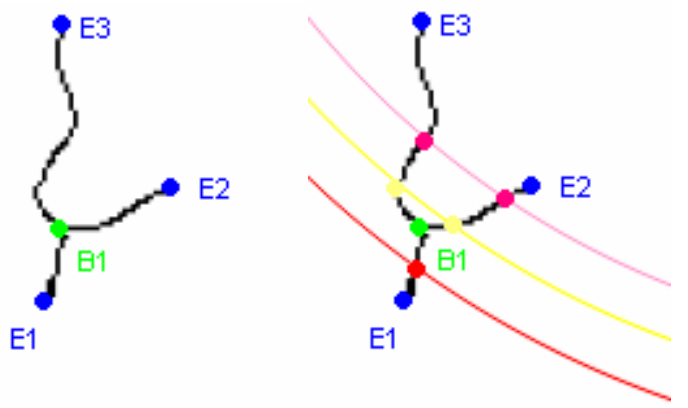

Fig. 9. The symbolic representation of the second step of feature extraction algorithm. 
The general rule for forming the second vector for each contour is presented below. First we store the number of endings, bifurcations and intersection points, and then we store all the coordinates of those points for all the extracted and traced contours. For $C$ contours in a given image we obtain:

$$
\begin{aligned}
F=\{ & \left\lfloor\left(N_{E}, N_{B}, N_{I}\right)\left(e_{1}, \cdots, e_{N_{E}}, b_{1}, \cdots, b_{N_{B}}, i_{1}, \cdots, i_{N_{I}}\right)\right\rfloor_{1} \cdots \\
& \left.\cdots\left\lfloor\left(N_{E}, N_{B}, N_{I}\right)\left(e_{1}, \cdots, e_{N_{E}}, b_{1}, \cdots, b_{N_{B}}, i_{1}, \cdots, i_{N_{I}}\right)\right\rfloor_{C}\right\}
\end{aligned}
$$

where:

$N_{E}$ - number of endings in each contour,

$N_{B}$ - number of bifurcations in each contour,

$N_{I}$ - number of points intersecting the circles,

$e$ - coordinates of endings,

$b$ - coordinates of bifurcations,

$i$ - coordinates of intersections in each contour.

\section{Classification}

For each image stored in the database we have two vectors $F_{r e f}$ and $V_{\text {ref }}$. For each input ear, we acquire many images under different angles to the camera.

The algorithm for recognition of an input image is following:

1. for the fixed number of circles, the feature vectors $V$ and $F$ of the input image are obtained

2. for each radius, we search the database feature vectors $V_{\text {ref }}$ that have the same number of intersections $l_{r}$ for the corresponding radiuses

3. the vectors with the number of intersections $\left(l_{r} \pm \delta\right)$ are also accepted, allowing the difference of $\delta$ pixel on each circle

4. in the next step we check if the difference within the distance sum $\sum d$ for all the extracted vectors is less than a certain threshold value

5. if none of the vectors $V_{\text {ref }}$ are found for the input image, the input image is rejected

6. if the number of intersecting points $l_{r}$ is accepted and the difference within the distance sum $\sum d$ is less than a certain threshold value we check the contour-topology vector $F$

7. we first search for the same triples $\left(N_{E}, N_{B}, N_{I}\right)$ of the input contour-topology vector $F$ with the reference contour vectors $F_{\text {ref }}$ 
8. then for the images with the same triples $\left(N_{E}, N_{B}, N_{I}\right)$ we check if the coordinates of the stored points are the same

9. if the corresponding coordinates of those vectors refer to the same points, the algorithm finds the winner of classification.

\section{Experimental Results and Future Work}

We perform our experiments on our own database of collected ear images. At the moment of writing, the database consists of over 240 images, but we are still adding more ear images of different type. For each person included in the experiments, we collected 2 left ear images, first with the camera perpendicular to the head and the second, with the camera within the specified angle of 30 degrees. Now, analogically to face recognition systems, we collect larger database of ear images, which contains 20 different views for each person ( 5 orientations, 2 scales, 2 illuminations).

We divided the database to several sets of images concerning their quality and degree of complexity. So far we have only experimented with images of very high quality and with the ideal conditions of recognition, without illumination changes (Figure 10). For such "easy" images from our database we obtained error-free recognition.
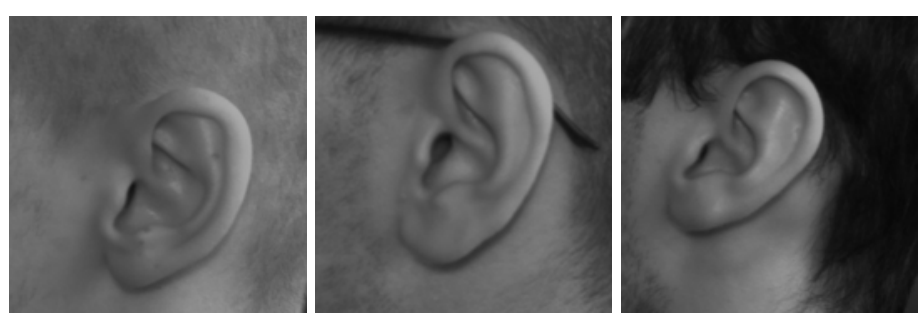

Fig. 10. Some examples of "easy" ear images from our database.

In further experiments we work with the "difficult" images and with the changing conditions of the image acquisition. In order to achieve satisfactory results with such complex images (Figure 11) we are improving the contour detection algorithm, so that long, straight line-contours of glasses and artificial contours of earrings and hair are eliminated before applying feature extraction algorithm. Moreover, we work on the algorithm selecting only the most significant ear contours. We eliminate contours that are short in comparison to the longest contour detected in the ear image.
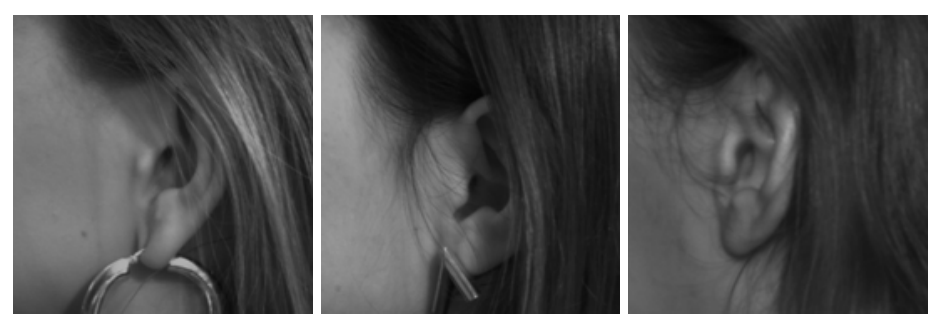

Fig. 11. Some examples of "difficult" ear images from our database.

We also think that the feature vectors should be enriched with more geometrical features in order to better distinguish ear identity. We are testing some new geometrical parameters describing shapes of ear contours and we compare their effectiveness in ear identification.

Moreover, we search for other than geometrical features describing ear images, such as energy and shape parameters. We try to discover, which features are the most significant in determining ear identity, so that we will be able to weight them properly in the process of building hybrid vectors of features of different types. 


\section{Conclusions.}

In the article we proposed a human identification method based on human ear images. We proposed invariant geometrical method in order to extract features needed to classification. First we perform contour detection algorithm, then coordinates normalization. Thanks to placing the centre of the new coordinates system in the centroid, our method is invariant to rotation, translation and scaling, which will allow RST queries. The centroid is also a key reference point in the feature extraction algorithm, which is divided into 2 steps. In the first step, we create circles centred in the centroid and we count the number of intersection points for each radius and the sum of all the distances between those points. All those points are stored in the first feature vector corresponding to the radiuses. In the second step, we use the created circles, but hereby we count the intersection points for each contour line. Moreover, while tracing the contour lines, we detect the characteristic points like endings and bifurcations. Together with the intersection points for each contour, we store them in the second feature vector corresponding to contour topology. Then we perform classification, basing on the simple comparison between the input image feature vectors, and all the vectors from the database. So far we have obtained very good results, however we still continue our research in order to improve our method and add more parameters to the feature vectors.

We believe that human ear is a perfect source of data for passive person identification in many applications. In a growing need for security in various public places, ear biometrics seem to be a good solution, since ears are visible and its images can be easily taken, even without the knowledge of the examined person. Then the robust feature extraction method can be used to determine personality of some individuals, for instance terrorists at the airports and stations. Access control to various buildings and crowd surveillance are among other possible applications.

Ear biometrics can be also used to enhance effectiveness of other well-known biometrics, by its implementation in multimodal systems. Since most of the methods have some drawbacks, recently, the idea of building multimodal (hybrid) biometrics systems is gaining lot of attention [11,16]. Due to its advantages, ear biometrics seem to be a good choice to support well known methods like voice, hand or face identification.

\section{References}

1. Ashbourn J., Biometrics - Advanced Identity Verification, Springer-Verlag 2000.

2. Beveridge J.R., She R., Draper B.A., Givens G.H., Parametric and Nonparametric Methods for the Statistical Evaluation of Human Id Algorithms, Workshop on Evaluation Methods in Computer Vision, 2001.

3. Bowman E., Everything You Need to Know about Biometrics, Technical Report, Identix Corporation, 2000.

4. Burge M., Burger W., Ear Biometrics, Johannes Kepler University, Linz, Austria 1999.

5. Burge M., Burger W., Ear Biometrics for Machine Vision, 21 Workshop of the Austrian Association for Pattern Recognition, Hallstatt, 1997.

6. Canny J., A Computational Approach to Edge Detection, IEEE Trans. on Pattern Analysis and Machine Intelligence, vol. 8, no. 6, 679-698, 1986.

7. Choraś M., Human Identification Based on Image Analysis - New Trends, Proc. Int. IEEE Workshop Signal Processing'03, pp. 111-116, Poznan 2003.

8. Choraś M., Human Ear Identification Based on Image Anlysis, in L. Rutkowski et al. (Eds): Artificial Inteligence and Soft Computing, ICAISC 2004, 688-693, LNAI 3070, Springer-Verlag 2004.

9. Choraś M., Feature Extraction Based on Contour Processing in Ear Biometrics, IEEE Workshop on Multimedia Communications and Services, MCS'04, 15-19, Cracow.

10. Danielsson P. E., Ye Q. Z., Rotation-Invariant Operators Applied to Enhancement of Fingerprints, Proc. $8^{\text {th }}$ ICPR, Rome 1988. 
11. Hong L, Jain A.K., Pankanti S., Can Multibiometrics Improve Performance?, Proc. of AutolD'99, 59-64, 1999.

12. Hoogstrate A.J., Heuvel van den H., Huyben E., Ear Identification Based on Surveillance Camera's Images, Netherlands Forensic Institute, 2000.

13. Hurley D.J., Nixon M.S., Carter J.N., Force Field Energy Functionals for Image Feature Extraction, Image and Vision Computing Journal, vol. 20, no. 5-6, 311-318, 2002.

14. Iannarelli A., Ear Identification, Forensic Identification Series, Paramont Publishing Company, California 1989.

15. Jain A., Bolle R., Pankanti S., Biometrics: Personal Identification in Networked Society, Kluwer Academic Publishers, 1999.

16. Jain A.K., Ross A., Multibiometric Systems, Comm. ACM, Special Issue on Multimodal Interfaces, vol. 47, no. 1, 34-40, 2004.

17. Jain L. C., Halici U., Hayashi I., Lee S. B., Tsutsui S., Intelligent Biometric Techniques in Fingerprint and Face Recognition, CRC Press International Series on Computational Intelligence, 1999.

18. Kouzani A.Z., He F., Sammut K., Towards Invariant Face Recognition, Journal of Information Sciences 123, Elsevier 2000.

19. Lai K., Chin R., Deformable Contours: Modeling and Extraction, IEEE Trans. on Pattern Analysis and Machine Intelligence, vol. 17, no. 11, 1084-1090, 1995.

20. Moreno B., Sanchez A., Velez J.F., On the Use of Outer Ear Images for Personal Identification in Security Applications, IEEE Conf. On Security Technology, 469-476, 1999.

21. Safar M., Shahabi C., Sun X., Image Retrieval By Shape: A Comparative Study, University of Southern California, November 1999.

22. Victor B., Bowyer K.W., Sarkar S., An Evaluation of Face and Ear Biometrics, Proc. of Intl. Conf. on Pattern Recognition, I: 429-432, 2002.

23. Zhang D., Automated Biometrics - Technologies and Systems, Kluwer Academic Publishers, 2000. 